\title{
Spontaneous haemarthrosis following thrombolytic therapy for myocardial infarction.
}

\author{
K.G. Oldroyd ${ }^{1}$, R.S. Hornung ${ }^{1}$, A.M. Jones ${ }^{2}$, N.P. Andrews ${ }^{2}$, P.T. Dawes ${ }^{2}$ and \\ P.H.M. Carson ${ }^{2}$
}

\author{
${ }^{1}$ Department of Medical Cardiology, Royal Infirmary, Glasgow G31 2ER and ${ }^{2}$ Departments of Cardiology \\ and Rheumatology, North Staffordshire Health District, UK.
}

\begin{abstract}
Summary: Two cases of haemarthrosis following thrombolytic therapy for acute myocardial infarction are described. Both patients had active pre-existing inflammatory disease in the affected joints at the time of presentation. This complication of thrombolytic therapy has not been previously reported.
\end{abstract}

\section{Introduction}

Thrombolytic therapy is now commonly administered to patients with acute myocardial infarction and, when given early enough, significantly reduces mortality. As its use increases, new and unexpected complications may occur.

\section{Case reports}

\section{Case 1}

A 63 year old previously well man presented as an emergency with a 3 hour episode of chest pain. He had undergone right knee menisectomy 35 years previously and 5 weeks before admission had experienced intermittent pain and swelling of his right knee and calf. He was taking no regular medications. Electrocardiography showed acute anterolateral infarction. Streptokinase (1.5 million units over 1 hour) and aspirin $(300 \mathrm{mg})$ were administered. Heparin (1000 units per hour intravenously) was added 2 hours after admission. About 11 hours later the patient noted increasing pain and swelling of his right knee and calf. On examination no bruising was seen. A provisional diagnosis of ruptured Baker's cyst was made. The following day, swelling and pain of the right knee and calf increased with the appearance of bruising. Heparin was discontinued, and aspiration of the knee yielded heavily blood stained fluid. The aspirate was sterile, did not clot and had a xanthochromic supernatant. Other investigations: kaolin cephalin clotting time (off heparin) 36 seconds (ratio 0.88 ), haemoglobin $13.8 \mathrm{~g} / \mathrm{dl}$, white

Correspondence: K.G. Oldroyd, M.R.C.P.

Accepted: 22 November 1989 cell count $12.8 \times 10^{9} / 1$, platelets $197 \times 10^{9} / 1$, ESR $12 \mathrm{~mm} / \mathrm{h}$, rheumatoid and antinuclear factors negative. The knee was splinted, and one further aspiration was required. After a period of rest and subsequent physiotherapy the patient made an uncomplicated recovery. X-ray of the knee showed patello-femoral osteoarthritis.

\section{Case 2}

A 68 year old woman presented as an emergency with a 2 hour history of chest pain and vomiting. In the previous 2 weeks she had experienced intermittent chest pain typical of angina pectoris. One year earlier she had developed pyoderma gangrenosum with an associated seronegative symmetrical erosive polyarthropathy mainly involving the elbows and knees. The skin lesion had responded to steroids but the arthropathy remained active. Regular medications were prednisolone $10 \mathrm{mg}$ on alternate days and co-proxamol as required. Electrocardiography showed acute anterior infarction. Streptokinase (1.5 million units over 1 hour) and aspirin $(150 \mathrm{mg})$ were administered. Heparin (1000 units per hour intravenously) was added 6 hours after admission. About 6 hours later, the patient noted increasing pain and swelling of the right elbow and left knee. On examination both joints were hot and swollen with effusions which had not been present on admission. No bruising was seen. The left knee was aspirated and $30 \mathrm{ml}$ of turbid fluid removed. By the next day extensive bruising had developed around the right elbow. Heparin was discontinued and aspiration of the right elbow yielded heavily blood stained fluid. Both aspirates were sterile with no crystals seen on polarising light microscopy. Other investigations: kaolin cephalin clotting time (on heparin) 84 
seconds (ratio 1.9), haemoglobin $11.4 \mathrm{~g} / \mathrm{dl}$, white cell count $13.6 \times 10^{9} / 1$, ESR $40 \mathrm{~mm} / \mathrm{h}$, antinuclear and rheumatoid factors negative. The elbow was splinted and after a period of rest, the haemarthrosis resolved satisfactory.

\section{Discussion}

Spontaneous haemarthrosis with subsequent joint damage is a recognized complication of oral anticoagulant therapy. Previously reported cases have often had pre-existing joint disease, and usually prothrombin times have been within the therapeutic range. ${ }^{1}$ In prospective studies ${ }^{2,3}$ on monitoring heparin therapy, spontaneous haemarthroses occurred in 2 out of a total of 443 patients, accounting for $6 \%$ of the major bleeding complications. It was also noted that there was no relationship between bleeding and either the heparin dosage or the activated partial thromboplastin time.

We have been unable to identify any reports of haemarthrosis following thrombolytic therapy and no reports have been received by the Committee on

\section{References}

1. Andes, W.A. \& Edmunds, J.O. Hemarthrosis and warfarin: joint destruction with anticoagulation. Thromb Haemost 1983, 49: $187-189$.

2. Basu, D., Gallus, A., Hirsh, J. \& Cade, J. A prospective study of the value of monitoring heparin treatment with the activated partial thromboplastin time. $N$ Engl J Med 1972, 287: 324-327.

3. Salzman, E.W., Deykin, D., Shapiro, R.M. \& Rosenberg, R. Management of heparin therapy: controlled prospective trial. N Engl J Med 1975, 292: 1046-1050. the Safety of Medicines. No haemarthroses were reported during the ISIS- 2 trial, ${ }^{4}$ which used the $\frac{2}{3}$ same dose of streptokinase as our patients received, $\frac{\mathbb{}}{\varnothing}$ and in which, a third received concurrent treatment $c$ with heparin (personal communication Dr R. Collins, Co-ordinator). Nonetheless, in our cases it is $\stackrel{\oplus}{\stackrel{D}{?}}$ difficult to implicate heparin alone and to dismiss $\bar{C}$ the potentiating role of fibrinolysis. If haemarthrosis does occur after thrombolytic therapy, any $\frac{\bar{\omega}}{\vec{D}}$ concurrently administered heparin should be dis- $\propto$ continued and the affected joint aspirated to minimize the risk of serious joint damage.

It is known that inappropriate anticoagulation in the pseudothrombophlebitis syndrome gives rise $\overrightarrow{\vec{\omega}}$ to significant morbidity. ${ }^{5} \mathrm{~A}$ history as in our first $\stackrel{\circ}{\circ}$ case suggesting intermittent synovial leakage 8 should alert the physician to this possible consequence of defibrination and may constitute a S $^{\circ}$ relative, though not in view of its proven benefits, an absolute contraindication to thrombolytic or therapy. We conclude that although the incidence $\dot{\infty}_{\infty}$ of serious joint bleeding after thrombolytic therapy

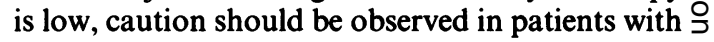
active joint disease.

4. ISIS-2 Collaborative Group. Randomised trial of intraveno streptokinase, oral aspirin, both, or neither among 17,18 suspected cases of suspected acute myocardial infarction: ISIS-2. Lancet 1988, ii: 349-360.

5. Tait, G.B.W., Bach, S. \& Dixon, A.St.J. Acute synovial ญै rupture: further observations. Ann Rheum Dis 1965, 24: 273-277. 